\title{
Numerical Simulation Study of Three-Dimensional Flow Field on Roots- Type Power Machine Based on Dynamic Mesh Technique
}

\author{
Xiao Yanjun*, Liu Yuxiang, Wang Dayuan and Li Chunxia \\ School of Mechanical Engineering, Hebei University of Technology, 300000, P.R. China
}

\begin{abstract}
Expander which drives an electromotor to generate electricity is the core of low-temperature waste heat recovery equipment. At present, domestic expanders on waste heat recovery system mostly arise from exploration, research and improvement on the existing models of screw expanders and scroll expanders, which have complex structures and high costs. For overcoming the shortcomings mentioned above, a new roots-type power machine is researched and designed. In this paper, the working fluid of low pressure waste heat is stimulated according to both the different operating conditions and the different intake and exhaust pressure and flow, and the changing process of internal flow field is simulated over the time period using dynamic mesh technique when the power machine is in rotating work. Besides, the pressure field, velocity field and graph of mass flow rate are analyzed with the simulation results, thus obtaining the conclusion of optimum operating conditions of the roots-type power machine to guide selection method in practice, that is, selecting appropriate roots-type power machines according to different types of waste heat in industrial production. These efforts can therefore provide strong theoretical guidance and foundation for the subsequent engineering practice of waste heat recovery system on roots-type power machine, and can have a profound impact on further recovery and utilization of low-grade energy.
\end{abstract}

Keywords: Dynamic mesh, industrial low-temperature waste heat, numerical simulation, roots-type power machine, the optimum operating conditions.

\section{INTRODUCTION}

Heat is a form of secondary energy, generated from combustible gas which is involved in various forms of primary energy used in the industrial processes, such as coal, oil, natural gas,etc. According to the temperature, it is divided into low-temperature waste heat, intermediatetemperature waste heat and high-temperature waste heat [1]. Waste Heat Resources are widely distributed in industrial production in about all walks of life having a wide variety. For example, the coal gas pressure differential energy evolving from top of blast furnaces in steel industry, coke oven gas, blast furnace gas, and converter gas generated in the production processes [2, 3]; In the cement industry, medium - low temperature exhaust gas at about $350{ }^{\circ} \mathrm{C}$ discharged from cement kilns accounts for about $30 \%$ of the total heat input [4]; In the non-ferrous metallurgy industry, the high temperature flue gas heat generated from kilns accounts for about half of the total heat in this industry [5]; In the production process of petrochemical industry, heat exchangers bring about large amount of steam or hot water [6]; Waste heat in the glass and building materials industry is mostly flue gas heat generated from kilns [7]. Waste heat resources have different characteristics according to different types and industries, which directly affect the design of technical solutions for waste heat recovery.

At present, domestic medium-high temperature cogeneration technology is dominated by the steam turbine power generation system consisting of waste heat boiler and

*Address correspondence to this author at the School of Mechanical Engineering, Hebei University of Technology, Tianjin, 300000, P.R. China; Tel: +86-13388007288; E-mail: x_yanjun2009@163.com a matching steam turbine. Moreover, the system above is widely used in iron and steel, metallurgy and other industries, but the applications are not widespread in the field of medium-low temperature waste heat. At present, in the field of low-temperature cogeneration technology, there are two main waste heat utilization systems, where the key equipments are scroll expander and screw expander [8-10]. Research in this area mostly involves exploration, research and improvement of the existing models such as strengthening seals, increasing lubrication, structural optimization design, etc. Expander is the key equipment in waste heat recovery system. With regard to the disadvantages of complex structure and high cost of the existing expanders, a new roots-type power machine is researched and designed. The new roots-type power machine has good geometric symmetry, and can run smoothly. Because of only two blades per rotor, it has a larger volume of the gas chamber and greater output power and torque. Therefore, in terms of the expanders of high power and high torque, the roots-type power machine is an optimum choice.

The roots-type power machine is a power machine which depends on fluid medium to push the rotating rotor to work. Its complex internal gas flow brings about many disadvantages if the methods of theoretical derivation and experimental verification are taken, such as long design cycles and high trial production costs, and it is difficult to obtain accurately the performance parameters of internal flow field. In this paper, the working fluid of low pressure waste heat is simulated according to both the different operating conditions and the different intake and exhaust pressure and flow, and the changing process of internal flow field is simulated over the time period using dynamic mesh 
technique when the power machine is in rotating work. Besides, the pressure field, velocity field and graph of mass flow rate are analyzed with the simulation results, thus obtaining the conclusion of optimum operating conditions of the roots-type power machine to guide selection method in practice, that is, selecting appropriate roots-type power machines according to different types of waste heat in industrial production.

\section{ESTABLISHMENT OF SIMULATION ENVIRON- MENT ON POWER MACHINE AT LOW TEMPE- RATURE CONDITIONS BASED ON FLUENT}

\subsection{Low-Temperature Working Conditions of Power Machine}

This paper mainly researched on low-temperature saturated steam generated from industrial production processes. In these researches, the pressure range of lowtemperature saturated steam is assumed to be at $0.2 \mathrm{MPa} \sim 1$ $\mathrm{MPa}$ and the corresponding temperature range at $134^{\circ} \mathrm{C} \sim 184^{\circ} \mathrm{C}$. The low-temperature saturated steam enters the working chamber from inlet of the power machine, and then pushes the rotating 8-shaped rotors to work. After acting, the waste steam is discharged from the exhaust port, as shown in Fig. (1). The rotors of power machine do rotational movement about the central axis under pushing of the fluid medium. Numerical simulation is needed to calculate force or torque on the rotors, thus identifying the motion law implemented on them, and analyzing the internal working performance of the power machine. In this paper, simulated calculation is conducted on recovery and utilization processes of low-temperature saturated steam, using numerical simulation methods of computational fluid dynamics and dynamic mesh technique, and then the working performance of power machine is analyzed at different low-temperature conditions, such as $0.2 \mathrm{MPa}, 0.3$ $\mathrm{MPa}, 0.4 \mathrm{MPa}, 0.5 \mathrm{MPa}, 0.6 \mathrm{MPa}, 0.7 \mathrm{MPa}, 0.8 \mathrm{MPa}, 0.9$ $\mathrm{MPa}$ and $1.0 \mathrm{MPa}$, respectively.

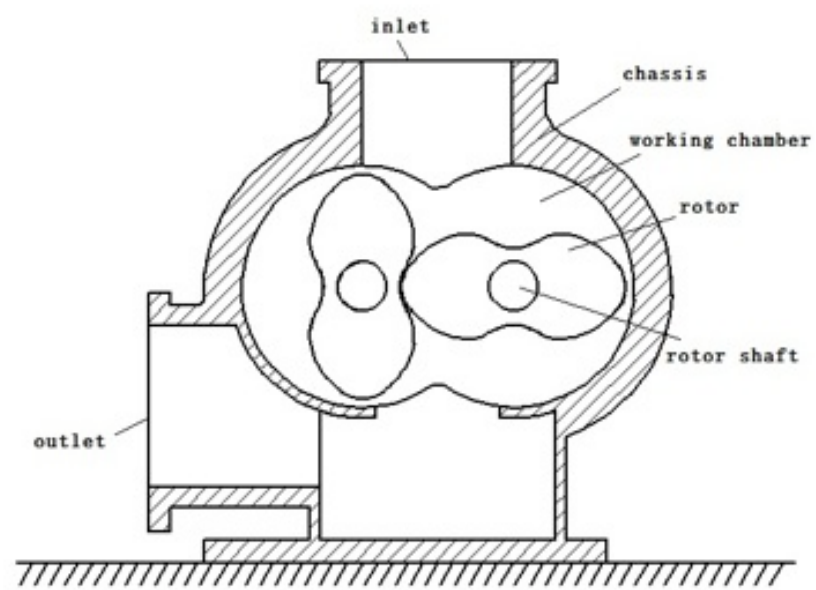

Fig. (1). Structure of roots-type power machine.

\subsection{Establishment of Watershed Model}

In this paper, the working process of roots-type power machine is simulated under pushing of saturated steam with certain pressure and temperature using the software named Fluent, so as to solve the interaction state between the rotor and the fluid when the roots-type power machine is in process of operating. First of all, geometric model of object of numerical simulation is established. In addition, according to the structural characteristics of roots-type power machine, the geometric model is divided into two parts: the fluid region; and the rotor. The fluid region is the area where the fluid flows through, including intake and exhaust passages and the working chamber composed by housing and rotors. 3D models in Solidworks are built, and the intake and exhaust ports of roots-type power machine are simplified for ease of calculation under the premise of not affecting the numerical simulation of flow field, as shown in Fig. (2). Furthermore, the geometric watershed model is established through Boolean operations, as shown in Fig. (3).

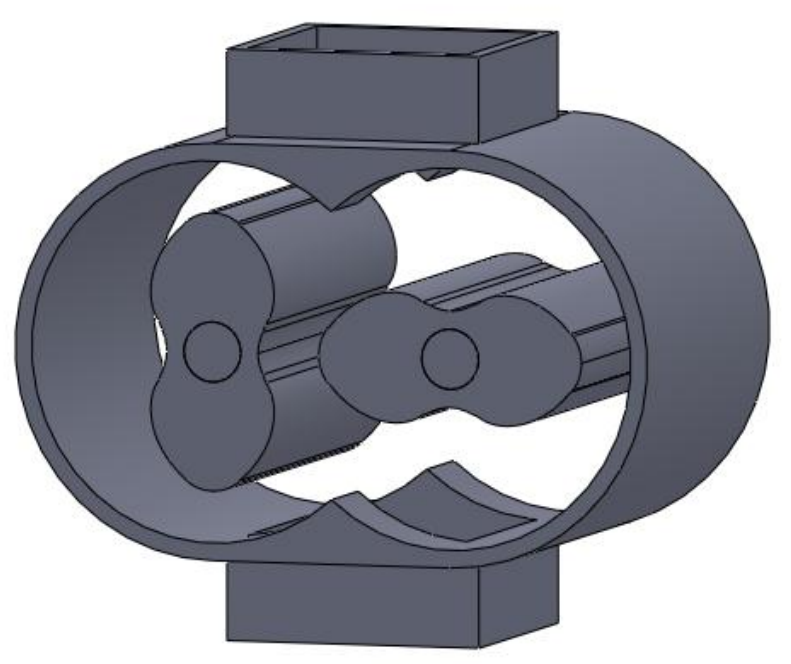

Fig. (2). 3D model of the roots-type power machine.

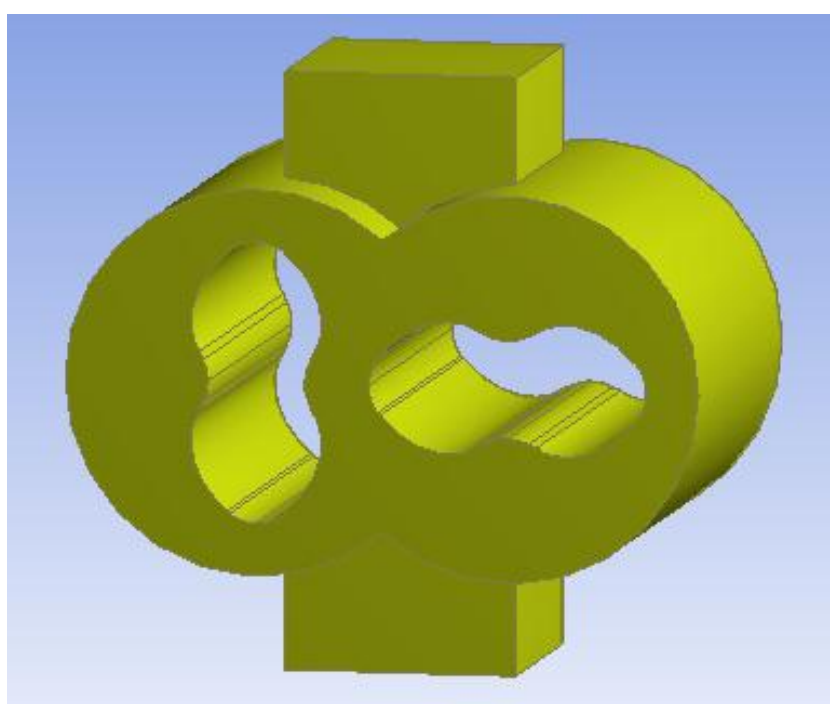

Fig. (3). Geometric watershed model of the roots-type engine.

Meshing is carried out in GAMBIT. Large deformation would occur in the calculation and higher quality for unit is needed in dynamic mesh computing. Considering the factors above, triangular prism-shaped meshes are applied into computational domain, so as to reduce the distortion rate of the mesh. Steam inlet is taken as an example to explain 
triangular prism-shaped meshes. The side of the inlet makes use of Quad units and map meshing (Fig. 4), cross section with Tri units and pave meshing (Fig. 5), and volume meshing with Hex/Wedge units and cooper meshing (Fig. 6). Fig. (7) shows an isometric view of a three-dimensional grid model and Fig. (8) presents a front view of a threedimensional grid model. As shown in Fig. (9), the total number of mesh units is 107,584 and the maximum cell skewness of meshes is less than 0.37. And the lower distortion rate of the unit is conducive to the realization of dynamic mesh.

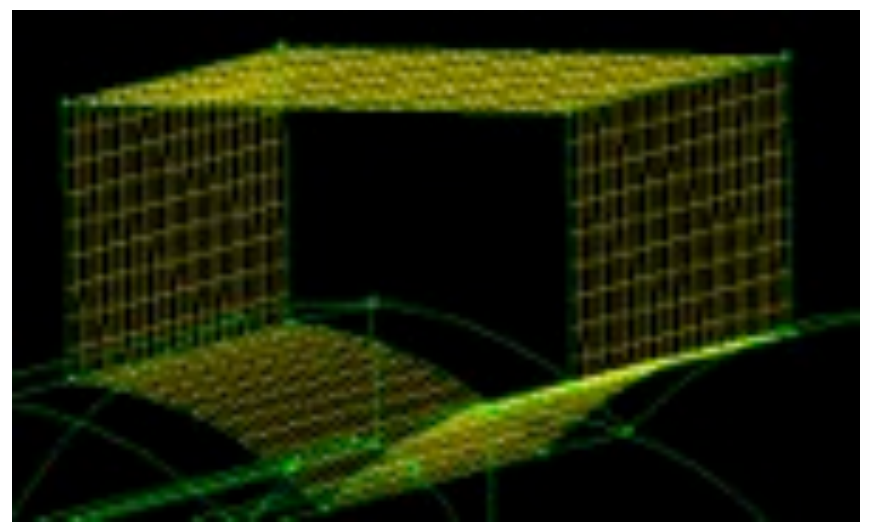

Fig. (4). Meshing the side.

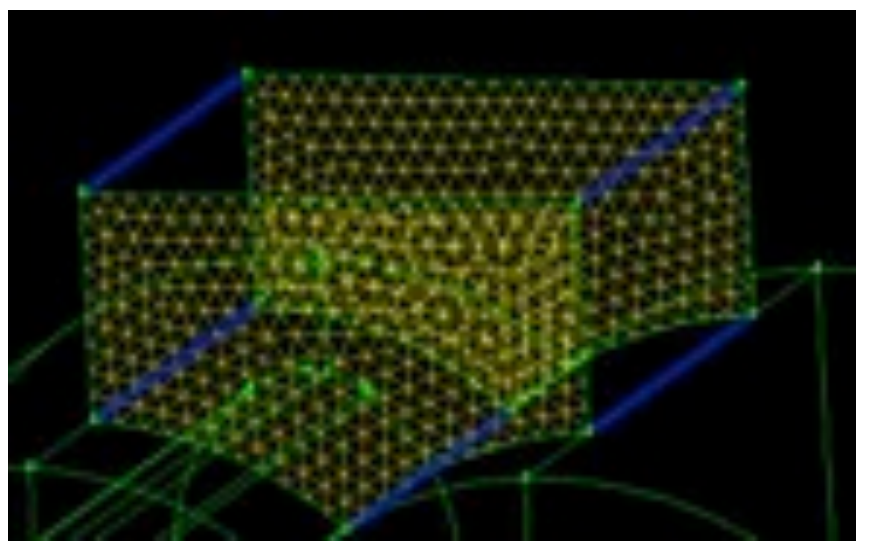

Fig. (5). Meshing the cross section.

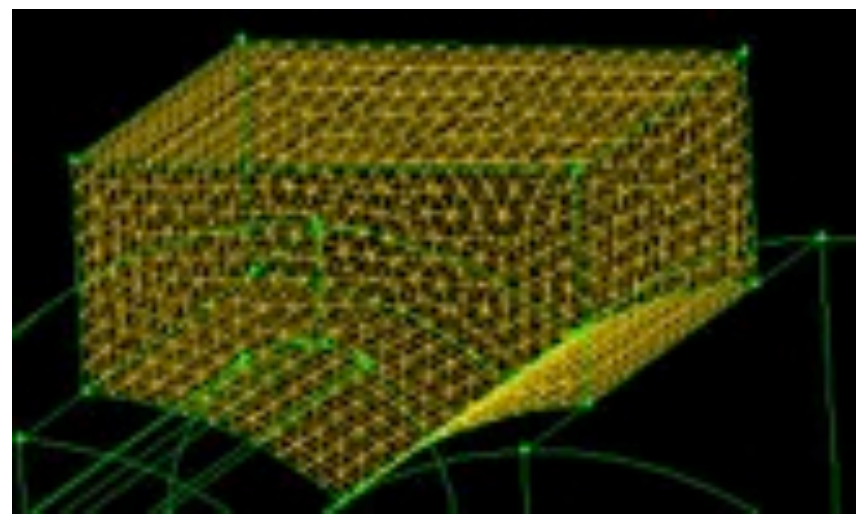

Fig. (6). Meshing volumes.

The boundary conditions are set after mesh generation: the inlet is mass flow inlet, the outlet is outflow, rotors on the left and right side are moving wall and the others are wall.

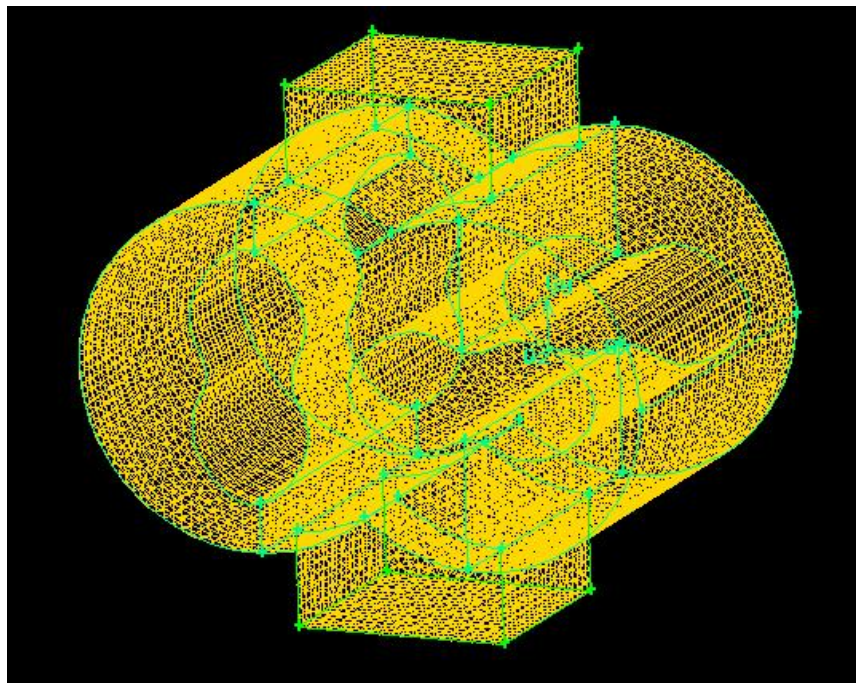

Fig. (7). the axonometric of meshes.

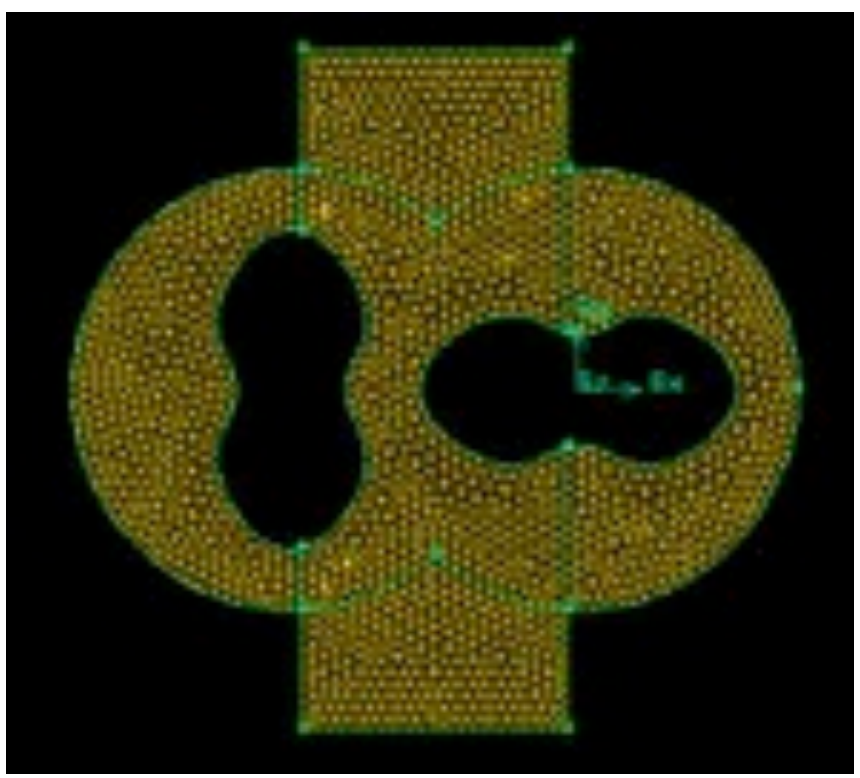

Fig. (8). The front view of meshes.

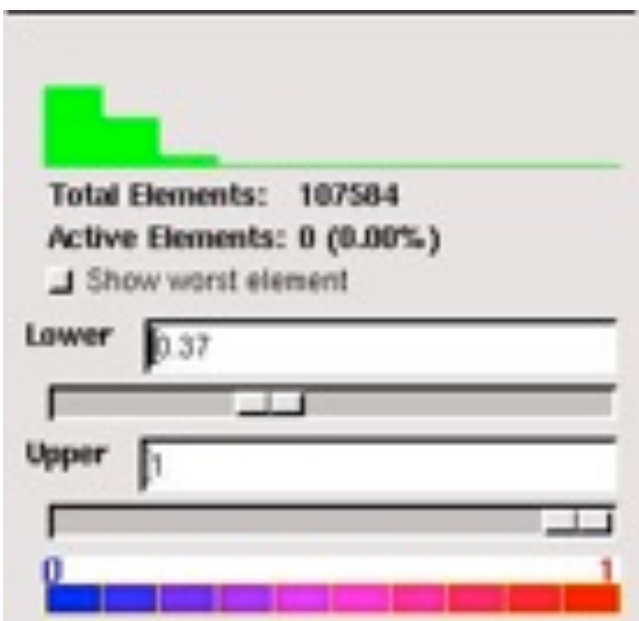

Fig. (9). Meshes skewnes. 


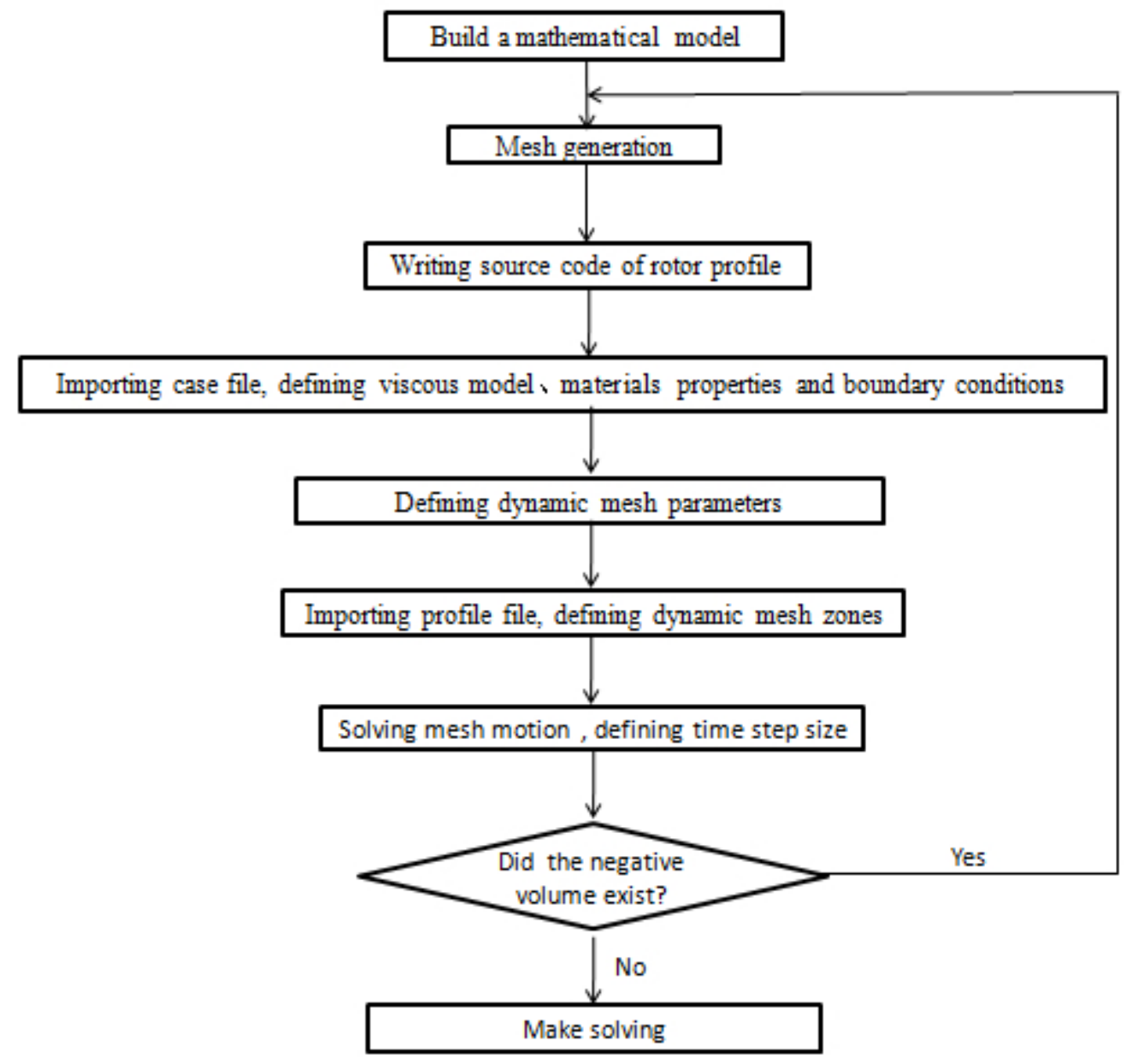

Fig. (10). Flowchart of parameters modification.

\subsection{Parameter Setting of Dynamic Mesh}

Working volume of the chamber in roots-type power machine changes over the time period along with the meshing rotation between the rotors. Numerical simulation of internal flow field on the power machine can only be achieved with dynamic mesh technique. The parameter setting of dynamic mesh is complex and prone to form negative volume, so it should be tried to improve the quality of the mesh before setting parameters to ensure the minimum distortion rate of the mesh. Moreover, attempts should be made to correct the parameters of dynamic mesh during the calculation. The flowchart of parameters modification is shown in Fig. (10).

In this paper, methods of smoothing and remeshing are applied into the dynamic mesh, and dynamic mesh model of $2.5 \mathrm{D}$ is selected. In the option of remeshing, the minimum length scale is $0.007 \mathrm{~m}$, maximum length scale is $0.012 \mathrm{~m}$, maximum face skewness is 0.6 , and size remesh interval is 1 . The profile is shown in Table 1.

\subsection{Selection of the Solver}

Based on different flow patterns, the fluid flow can be divided into laminar, transitional and turbulent flow $[11,12]$. Turbulent flow is very irregular and unstable, and the fluid particles exchange mass, momentum and kinetic energy by fluctuating, so the airflow in roots-type power machine is turbulent flow. The Reynolds number of roots-type power machine is higher, so the unsteady k-e model is selected as its turbulence model. Considering the convergence and convergence speed, an implicit solver is adopted based on pressure. The ideal air is chosen as the working medium, and the air follows the laws of conservation of mass, momentum and energy in the flow process. PISO is applied to the solving of continuity and momentum and PRESTO is adopted to calculate the pressure and upwind discretization of Second-order accuracy to solve the other problems.

Table 1: Dynamic mesh profile file of rotor.

\begin{tabular}{|c|c|c|}
\hline Name & Left Rotor & Right Rotor \\
\hline \hline \multirow{3}{*}{ Program code } & $\begin{array}{c}\text { (movingwall left 3 point) } \\
\text { (time 0 1 60) }\end{array}$ & $\begin{array}{c}\text { (movingwall_right 3 point) } \\
\text { (time 0 1 60) }\end{array}$ \\
& $\begin{array}{c}\text { (omega_z 157 157 157) } \\
\text { (omega_z -157 -157 -157) }\end{array}$ \\
\hline
\end{tabular}

\section{MOTION SIMULATION RESULTS AND ANALYSIS OF THE POWER MACHINE IN LOW- TEMPERATURE CONDITIONS}

It takes $0.04 \mathrm{~s}$ to turn a circle for the rotor and $0.0001 \mathrm{~s}$ is chosen as the time step size. In this paper, simulation and 
analysis are conducted on the following nine working conditions in order to analyze the internal flow situation of the power machine: condition $1(0.2 \mathrm{MPa})$, condition $2(0.3$ $\mathrm{MPa})$, condition $3(0.4 \mathrm{MPa})$, condition $4(0.5 \mathrm{MPa})$, condition $5(0.6 \mathrm{MPa})$, condition $6(0.7 \mathrm{MPa})$, condition 7 $(0.8 \mathrm{MPa})$, condition $8(0.9 \mathrm{MPa})$ and condition $9(1.0 \mathrm{MPa})$. During simulation and analysis, the performance of power machine is analyzed in different working conditions under the premise that the flow of the inlet remains constant. The simulation results of the three cases among them are listed below.

\subsection{Results and Analysis of Pressure Contours}

Fig. (11) shows the pressure contours of the results of motion simulation and the velocity vector diagram of some sections in condition 1 , in which the rotor rotates to 45 degrees, 90 degrees, 135 degrees and 180 degrees.

Based on the pressure contours, following conclusions can be drawn: 1) The red region and the blue region appear alternately on the boundary of the engaging portions of the two rotors, namely high and low pressure. The reason is that the two rotors extrude the working medium of the engaging portions in engagement process, which makes the instantaneous pressure of this position increase sharply and then the red region appears; with the end of rotor meshing, the space with the compressed gas expands greatly and the airflow in the region close to the outlet is discharged rapidly, which makes the instantaneous pressure of this position reduced gradually and then the blue region appears. With the

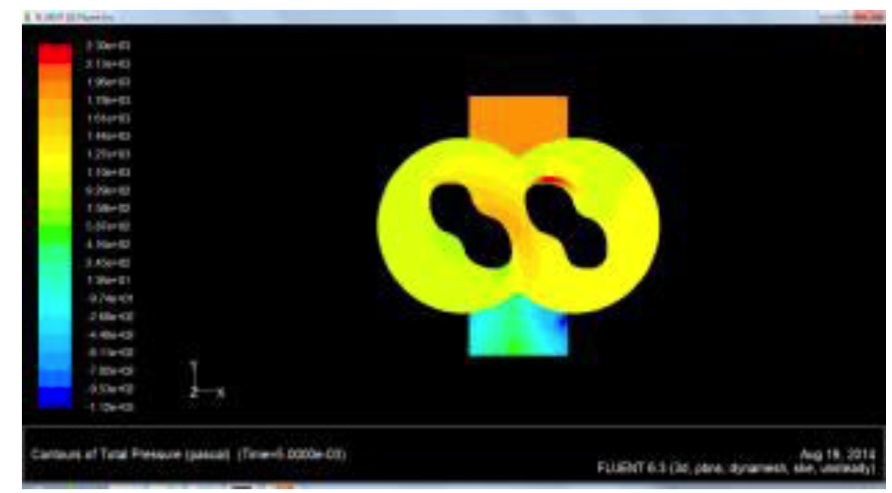

(a) $45^{\circ}$

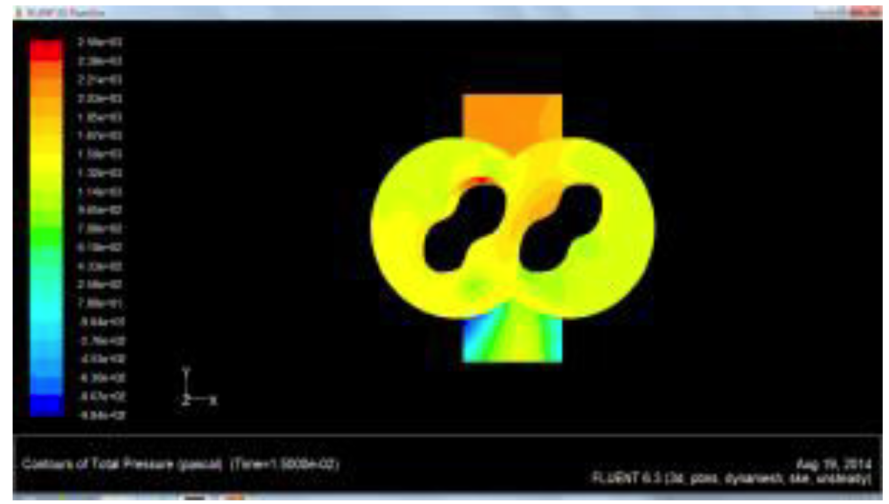

(c) $135^{\circ}$ high-speed rotation of the rotor, high and low pressure appear alternately in the process and the rotor is subjected to pressure shock, which becomes the main reason for the damage of rotors. 2) When the rotor rotates to 90 degrees and 180 degrees, the green area appears in the right volume. This is because the volume and discharge port are connected at this moment and the gas is rapidly excreted, which results in the reduction of pressure and the emergence of green area. 3) The pressure distribution of these working conditions is basically the same, but with the changing of working conditions, the maximum and minimum values of the speed in pressure contours change. The distribution of the green region in the right area of condition $8(0.9 \mathrm{MPa})$ and $9(1.0$ $\mathrm{MPa})$ is symmetrical and at the same time, the pressure does not exceed the maximum allowed pressure, as shown in Fig. (12).

\subsection{Results and Analysis of the Velocity Vector Diagram}

Fig. (13) shows pressure contours of the results of motion simulation and the velocity vector diagram of some sections in condition 1 , in which the rotor rotates to 45 degrees, 90 degrees, 135 degrees and 180 degrees.

From the velocity vector diagram, following conclusions can be drawn: 1) The flow rate of the air of inlet is much higher than that of the outlet when the rotor starts to spin, and eddy current phenomenon generates at both sides of the adjacent parts between vent and casing wall. However, as the rotor continues rotating and the flow rate of the vent increases, the eddy current phenomenon at both sides

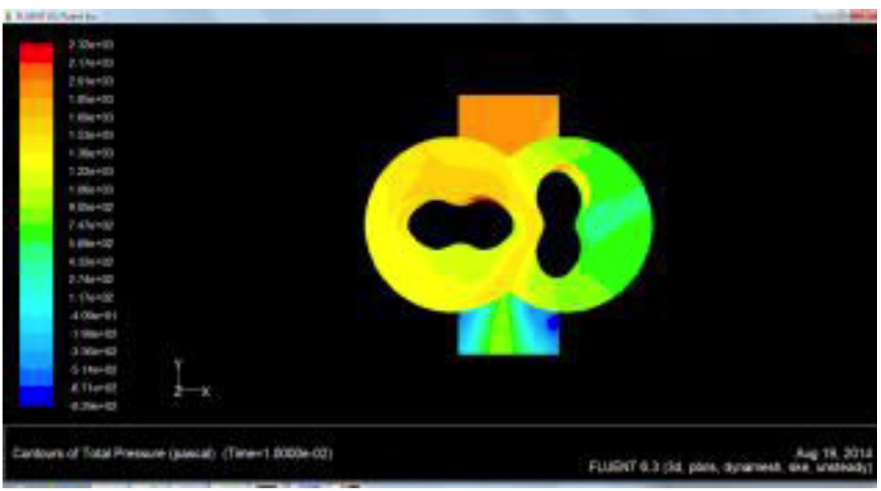

(b) $90^{\circ}$

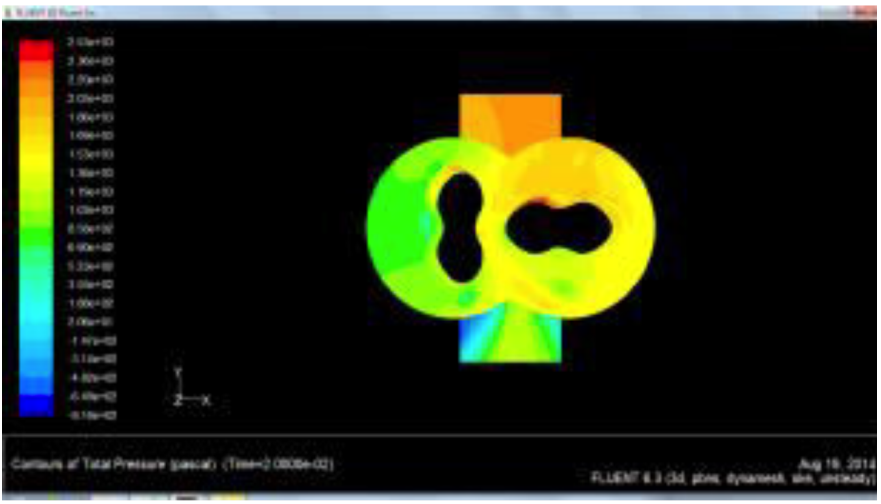

(d) $180^{\circ}$

Fig. (11). Total pressure contours of condition 1(0.2 MPa). 

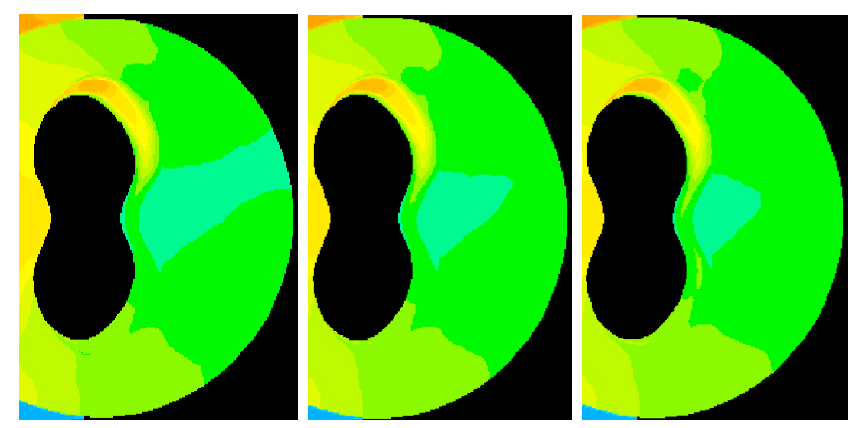

(a) condition 1

(b) condition 2

(c) condition 3
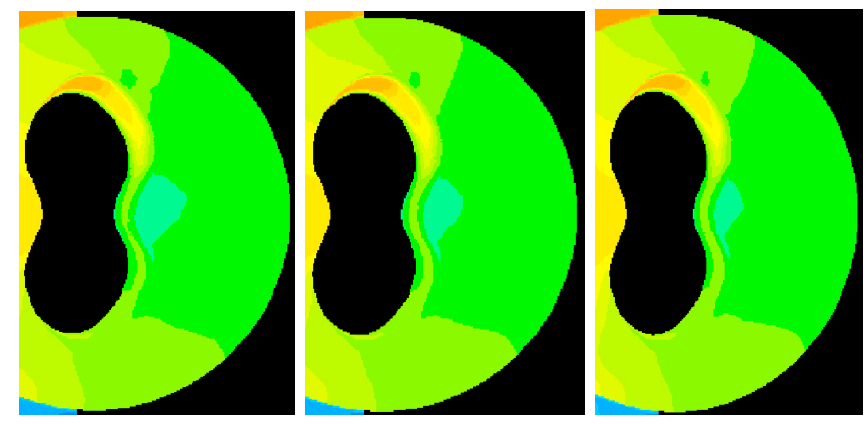

(d) condition 4

(e) condition 5

(f) condition 6
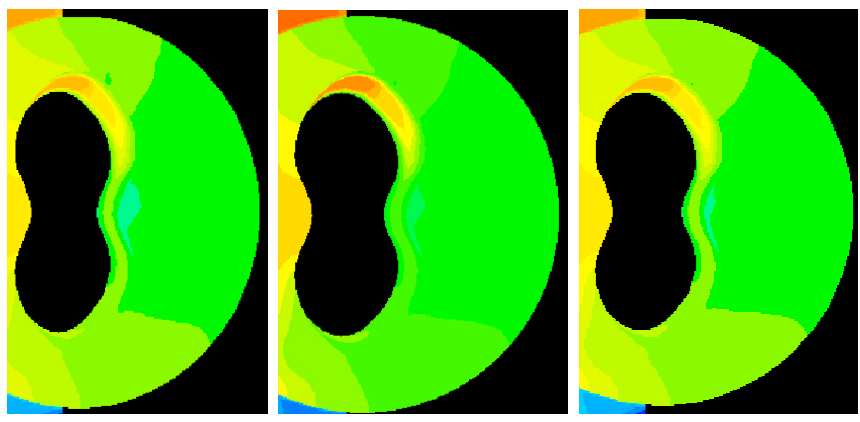

(g) condition 7

(h) condition 8

(i) condition 9

Fig. (12). Enlarged total pressure contours of rotor rotating $90^{\circ}$ in $1-9$ conditions.

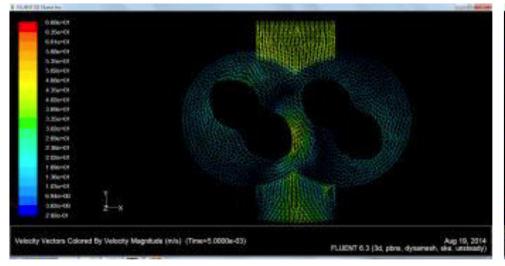

(a) $45^{\circ}$

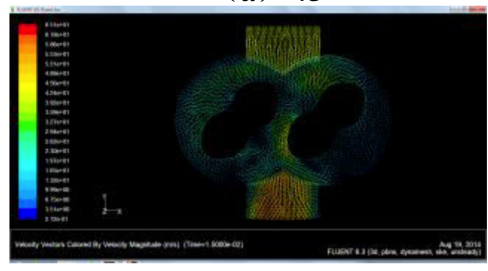

(c) $135^{\circ}$

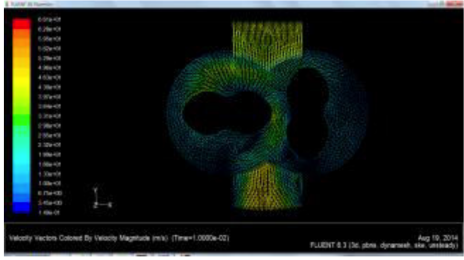

(b) $90^{\circ}$

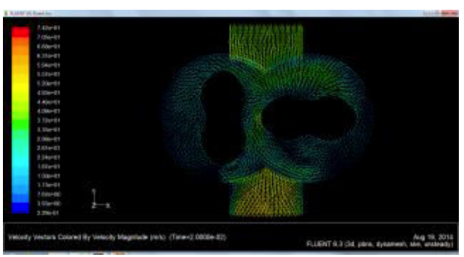

(d) $180^{\circ}$

Fig. (13). Velocity vector diagram of condition $1(0.2 \mathrm{MPa})$. 

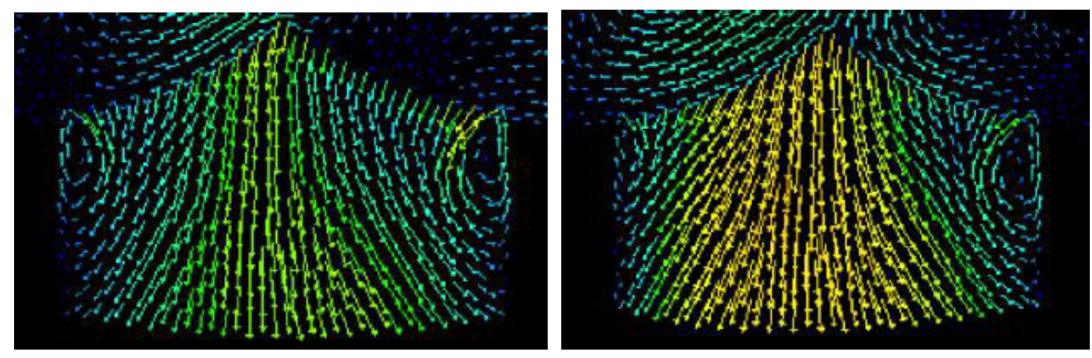

(a) $45^{\circ}$

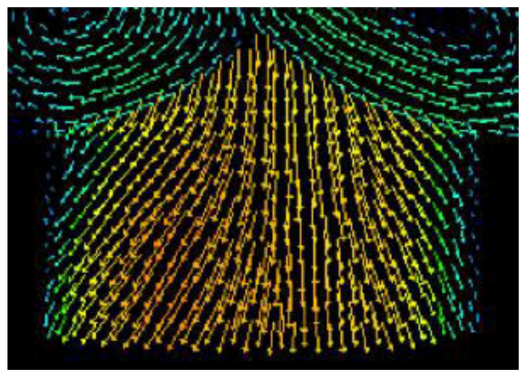

(c) $135^{\circ}$ (b) $90^{\circ}$

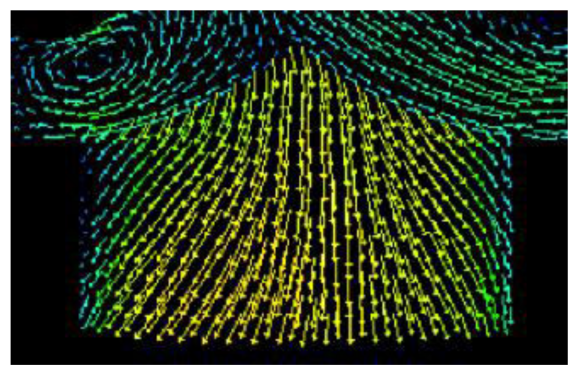

Fig. (14). Enlarged velocity diagram of inlet.

of the vent decreases gradually until it disappears, as shown in Fig. (14). The main cause of this process is that, when the velocity of the gas from the vent beside the casing wall is low, the airflow here mixes with the high-speed airflow of the vent and then eddy current phenomenon is generated. So with the increase of velocity of flow in outlet, eddy current gradually decreases until it finally disappearsing. 2) With the rotation of the rotor, two small vortexes are formed gradually close to the wrap of two rotors in the side of the vent. Moreover, through the simulation, the whole process of how the vortex forms can be observed clearly, as shown in Fig. (15). This is because the two rotors in the working chamber rotate in opposite directions at the same time and then eddy current phenomenon is generated owing to different directions of the internal airflow. At the same time, with the increasing flow rate of the vent, the flow direction of the air is more complex and the eddy current phenomenon appears in the side of the vent. 3) The velocity distribution of all working conditions is basically the same, but with the change of the conditions, the maximum and minimum speeds in the velocity vector diagram also change accordingly.

\subsection{Results and Analysis of the Mass Flow Graph}

This study uses the method of monitoring mass flow to judge convergence of results and the mass flow rate of inlet is set as $1.39 \mathrm{Kg} / \mathrm{s}$. The results can be obtained by comparing the mass flow graphs (Fig. 16) of 9 working conditions as shown: 1) The mass flow rate of different working conditions fluctuates at $1.39 \mathrm{Kg} / \mathrm{s}$ ups and downs and the error is very small, which further proves the accuracy of the analysis results. 2) By comparison, the fluctuations of mass flow rate in condition $1(0.2 \mathrm{MPa})$ and condition $8(0.9 \mathrm{Mpa})$ are the smallest.

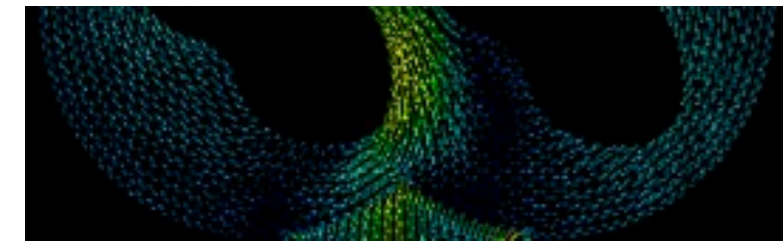

(a) $45^{\circ}$

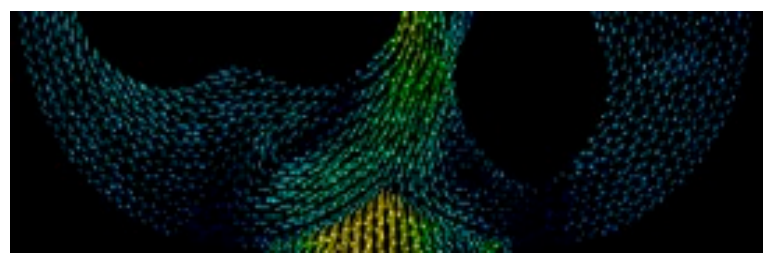

(b) $90^{\circ}$

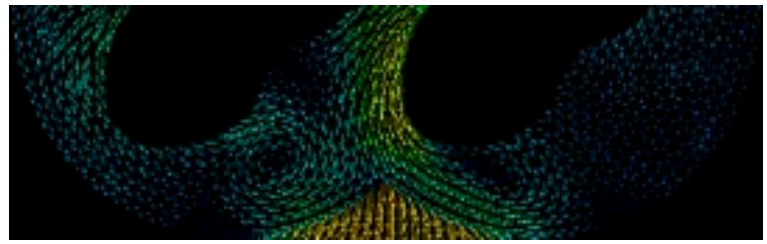

(c) $135^{\circ}$

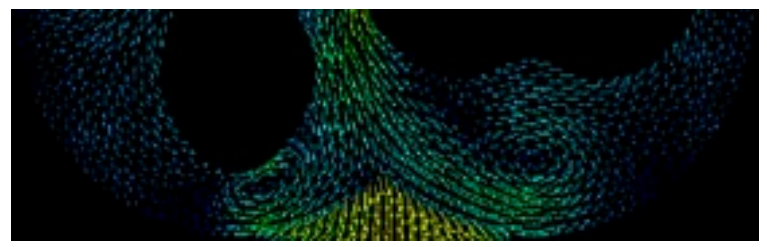

(d) $180^{\circ}$

Fig. (15). Enlarged velocity diagram of a part of roots-type engine. 


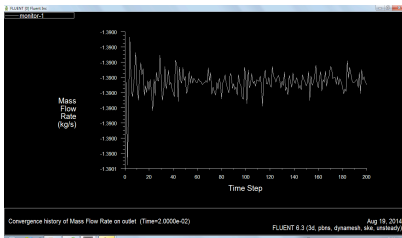

(a) condition 1

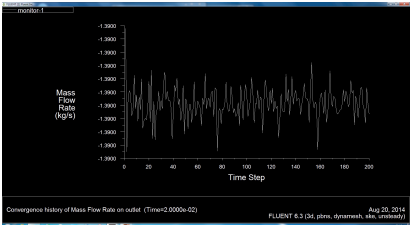

(c) condition 3

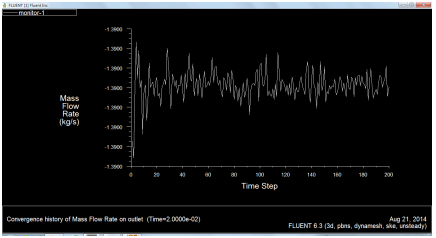

(e) condition 5

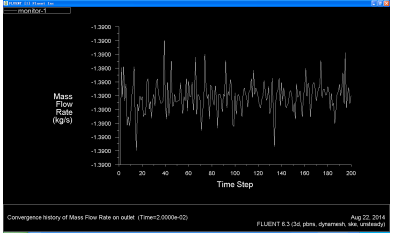

(g) condition 7

(h) condition 8

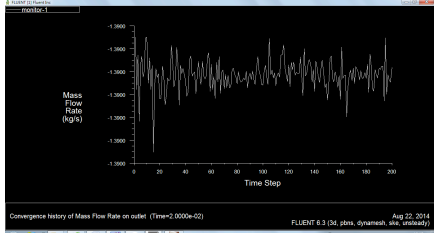

(i) condition 9

Fig. (16). Mass flow graphs in 1-9 conditions.

The roots-type power machine outputs the mechanical energy by means of fluid medium driving the rotors to work. Through analysis of the simulation results mentioned above and taking the pressure field, velocity field and mass flow graph into consideration, the best working condition can be obtained as: the impact force from fluid is within allowable range and running speed is stable. The corresponding selection principles choose the working conditions in which the boundary velocity of rotors is distributed evenly and fluctuations of the mass flow are small under the premise of the rotors within allowed pressure. The condition $8(0.9$ $\mathrm{Mpa}$ ) of roots-type power machine includes maximum stress $2820 \mathrm{pa}$, maximum speed $84.5 \mathrm{~m} / \mathrm{s}$ and mass flow rate fluctuating in $1.39 \mathrm{Kg} / \mathrm{s}$ ups and downs, which is a better working condition.

\section{CONCLUSION}

With the operating mode of optimum working conditions, the waste heat generated in industrial processes can be effectively utilized, which plays an important role in ensuring the recovery devices to operate safely and efficiently. In this paper, the changes of three-dimensional internal flow field were simulated in different conditions and the pressure field, velocity field and regulations of mass flow rate were shown clearly with the help of the fluid simulation software-Fluent and dynamic mesh technique. By comparing the differences of the simulations in different operating conditions, the optimum operating conditions of the rootstype power machine can be drawn to guide selection method in practice that is, selecting appropriate roots-type power machines according to different types of waste heat in industrial production.

\section{CONFLICT OF INTEREST}

Financial contributions to the work being reported should be clearly acknowledged, as should any potential conflict of interest.

\section{ACKNOWLEDGEMENTS}

The authors would like to thank the reviewers for their valuable comments, which have been utilized to improve the paper.

\section{REFERENCES}

[6] W. Dai, and H. Yin, "Optimal multi-period operational planning for steam power system of petrochemical industry with consideration of environmental costs," Acta Petrolei Sinica, vol. 26, no. 3, pp. 449-455, 2010.

[7] L. Li, "Discussion on energy saving and emission reduction of building material industry in the trend of low-carbon economy," Cement Engineering, vol. 3, pp. 1-8, 2010.

[8] B. Cao, and W. Li, "Analysis of dual-cycle low-temperature waste heat recovery system with screw expander," Journal of Tianjin University, vol. 43, no. 4, pp. 310-314, 2010.

[9] G. Liu, Y. Zhao, and L. Li, "Simulation of scroll expander used in low-temperature waste heat recovery system," Journal of Xian Jiao Tong University, vol. 43, no. 7, pp. 89-91, 2009. 
[10] N. Hayakawa, Y. Wakazono, T. Kato, and Y. Suzuoki, "Energy consumption in industries by cascade use of waste energy," IEEE Transactions on Energy Conversion, vol. 14, no. 3, pp. 795-801, 1999.

[11] A. Kovacevic, "Boundary adaptation in grid generation for CFD analysis of screw compressors," International Journal for
Numerical Methods in Engineering, vol. 64, no. 6, pp. 401-426, 2005.

[12] Z. Liu, L. Xu, and X. Zhao, "Unsteady numerical simulation and analysis of the flow fluctuation in the roots blower," Journal of Aerospace Power, vol. 22, no. 3, pp. 401-405, 2007.

(C) Yanjun et al.; Licensee Bentham Open

This is an open access article licensed under the terms of the Creative Commons Attribution Non-Commercial License (http://creativecommons.org/licenses/by-nc/4.0/) which permits unrestricted, non-commercial use, distribution and reproduction in any medium, provided the work is properly cited 